\title{
Synthesis and Evaluation of Antituberculosis Activity of Substituted 2,7-Dimethylimidazo [1,2-a]Pyridine-3-Carboxamide Derivatives
}

\author{
Bhagwat Jadhav ${ }^{1}$, R. Kenny ${ }^{2}$, Y. Nivid ${ }^{3}$, Mustapha Mandewale, Ramesh Yamgar $^{2 *}$ \\ ${ }^{1}$ P.G. and Research Centre, Department of Chemistry, Government of Maharashtra, Ismail Yusuf College of Arts, Science and Commerce, \\ Mumbai, India \\ ${ }^{2}$ Department of Chemistry, Chikitsak Samuha's Patkar-Varde College of Arts, Science and Commerce, Mumbai, India \\ ${ }^{3}$ Terna Medical College, Mumbai, India \\ Email:*rameshyamgar@gmail.com
}

How to cite this paper: Jadhav, B., Kenny, R., Nivid, Y., Mandewale, M. and Yamgar, R. (2016) Synthesis and Evaluation of Antituberculosis Activity of Substituted 2,7-Dimethylimidazo[1,2-a]Pyridine-3-Carboxamide Derivatives. Open Journal of Medicinal Chemistry, 6, 59-69.

http://dx.doi.org/10.4236/ojmc.2016.64006

Received: October 15, 2016

Accepted: December 26, 2016

Published: December 29, 2016

Copyright $\odot 2016$ by authors and Scientific Research Publishing Inc. This work is licensed under the Creative Commons Attribution International License (CC BY 4.0).

http://creativecommons.org/licenses/by/4.0/

\begin{abstract}
A series of substituted 2,7-dimethylimidazo[1,2-a]pyridine-3-carboxamides derivatives $\mathbf{5 a}-\mathbf{5 m}$ were synthesized through multi-step reactions. To achieve the synthesis of the desired compounds monobromo and dibromo substituted 2-amino- $\gamma$-picoline was reacted with ethyl 2-chloroacetoacetate. The crude ethyl ester subjected to hydrolysis in presence of lithium hydroxide to get $\mathbf{2 a}$ and $\mathbf{2 b}$, with imidazo[1,2-a]pyridine-3-carboxylic acid to get $\mathbf{3 a - 3} \mathbf{b}$, on treatment with substituted amines $\mathbf{4 a - 4} \mathbf{g}$ to get desired product $5 \mathrm{a}-5 \mathrm{~m}$ in presence of EDCI and HOBt. The substituted imidazo[1,2-a]pyridine-3-carboxamides are characterized by FTIR, ${ }^{1} \mathrm{H}-\mathrm{NMR},{ }^{13} \mathrm{C}-\mathrm{NMR}$ and mass spectra. These newly synthesized compounds were tested in vitro for their antimycobacterial activity. The preliminary results of antituberculosis study showed that most of the synthesized compounds $5 \mathrm{a}-5 \mathrm{~m}$ demonstrated moderate to good antituberculosis activity. Among the tested compounds $\mathbf{5 b}, \mathbf{5 d}$ and $\mathbf{5 e}$ were found to be the most active with minimum inhibitory concentration (MIC) of $12.5 \mu \mathrm{g} / \mathrm{mL}$ against Mycobacterium tuberculosis (H37 RV strain) ATCC No-27294.
\end{abstract}

\section{Keywords}

Carboxamides, Imidazo[1,2-a]Pyridine, Tuberculosis

\section{Introduction}

The imidazo[1,2-a]pyridines are found to possess various potential biological activities. A large number of substituted imidazo[1,2-a]pyridines have been synthesized and 
tested for various biological activities such as antiulcer [1], antibacterial [2], antimicrobial [3], antifungal [4], and antiviral [5] [6] agents. Various imidazo fused heterocycles with aryloxy alkylamines side chain including imidazo[1,2-a]pyridines act as calcium channel blockers or as local anaesthetics [7]. Byth and co-workers [8] have synthesized various pyrimidine sulfonamide substituted imidazo[1,2-a]pyridine as cyclindependent kinase (CDK) inhibitors which led to the identification of potent and selective inhibitors of CDK2 and CDK1. Several 8-arylimidazo[1,2-a]pyridines have been synthesized and evaluated as cardiotonic agents [9]. Some highly substituted imidazo[1,2-a]pyridines have been substituted as gastric antisecretory agents useful as gastric antiulcer agents [10]. Zhonghui Lu, Gregory R. Ott. and co-worker [11] [12] have synthesized a series of 2,3-substituted imidazo[1,2-a]pyridine derivatives which resemble $(3 S, 4 S)$ pyrrolidine- $N$-hydroxy-3-carboxamide and $(3 R, 4 R)$ pyran- $N$-hydroxy-3-carboxamide has been reported to have potent selectivity against tumor necrosis factor converting enzyme (TACE). The synthesis and tuberculosis activity of various substituted imidazo[1,2-a]pyridine-3-carboxamides have been reported [13]. Katherine A. Abrahams et al. [14] described the synthesis and biological activity of imidazo[1,2-a] pyridine-3-carboxamides against Mycobacterium tuberculosis. Garrett C. Moraski et al. synthesized a series of nine 2,7-dimethylimidazo[1,2-a]pyridine-3-car- boxamides and one 2,6-dime-thylimidazo[1,2-a]pyrimidine-3-carboxamide and evaluated for their in vitro antituberculosis activity against replicating, nonreplicating, multi- and extensive drug resistant Mtb strains [15]. Linhu Li et al. [16] have developed the synthesis and antituberculosis activity of various imidazo[1,2-a]pyridine-3-car- boxamide hybrids with extended amine functionality. Recently Samala G. et al. [17] synthesized 2-methylimidazo[1,2-a]-pyridine-3-carboxamides as Mycobacterium tuberculosis Pantothenate synthetase inhibitors.

In continuation of our research for new antimicrobial agents [18] [19] [20] [21] [22], we have undertaken research studies on synthesis and biological screening of some new imidazo[1,2-a]pyridine derivatives.

\section{Materials and Methods}

Melting points of compounds were determined in open capillary tubes in silicon oil bath using a Veego melting point apparatus and are uncorrected. ${ }^{1} \mathrm{H}-\mathrm{NMR}$ and ${ }^{13} \mathrm{C}$-NMR spectra were acquired on Bruker (400 MHz), chemical shifts were given on the delta scale as parts per million (ppm) with tetramethylsilane (TMS) as the internal standard. Electron impact ionization mass spectra were recorded on Agilent Technologies 5975C MSD detector at $70 \mathrm{eV}$. Infrared spectra were measured with $\mathrm{KBr}$ pellet on a FTIR-7600 Lambda Scientific Pvt. Ltd. in the range $4000-400 \mathrm{~cm}^{-1}$. Thin layer chromatography (TLC) was performed on silica F254 coated aluminum plates (Merck) as adsorbent and the spots were visualized using ultraviolet light or iodine chamber.

\section{Chemistry}

We synthesized imidazo[1,2-a]pyridine-3-carboxamide derivatives and evaluated them 
for Antituberculosis Activity. Figure 1 envisages the schematic representation for the synthesis of (un)substituted 2,7-dimethylimidazo[1,2-a]pyridine-3-carboxamides (5a$5 \mathrm{~m})$. For the synthesis of the desired molecules, mono bromo and dibromo substituted 2-amino- $\gamma$-picoline (1a-1b) were reacted with ethyl 2-chloroacetoacetate in presence of base to give ethyl imidazo[1,2-a]pyridine-3-carboxylates (2a-2b). These esters were then hydrolized to give imidazo[1,2-a]pyridine-3-carboxylic acids (3a-3b). These acid derivatives were treated with (un)substituted anilines (4a-4f) using hydroxybenzotriazole (HOBt) and EDCI as coupling agent to afford (un)substituted 2,7-di-methylimidazo[1,2-a]pyridine-3-carboxamides (5a-5m).

The FT-IR spectrum of hydrazone $5 \mathrm{a}$, as a representative example, showed strong absorption bands at 1654 and $1595 \mathrm{~cm}^{-1}$ due to amide, $\mathrm{C}=\mathrm{O}$ stretching and $\mathrm{N}-\mathrm{H}$ bending respectively. The broad peak at $3138 \mathrm{~cm}^{-1}$ is attributed to amide $\mathrm{N}-\mathrm{H}$ stretching. Its ${ }^{1} \mathrm{H}-\mathrm{NMR}$ spectrum revealed, in addition to expected aromatic signals, three singlets at $\delta$ $2.69,2.87,9.67 \mathrm{ppm}$ are assignable to the two methyl groups and amide proton $(-\mathrm{NH}-\mathrm{C}=\mathrm{O})$, respectively. In addition, the ${ }^{13} \mathrm{C}-\mathrm{NMR}$ spectrum of $5 \mathrm{a}$ displayed characteristic peak at $\delta 158.5 \mathrm{ppm}$ assignable to carbonyl carbon of amide. Moreover the EIMS spectrum of 5 a revealed molecular ion peak at $\mathrm{m} / \mathrm{z} 423.9(\mathrm{M}+)$ corresponding to molecular formula $\left[\mathrm{C}_{16} \mathrm{H}_{13} \mathrm{Br}_{2} \mathrm{~N}_{3} \mathrm{O}\right]$. In a similar manner, compounds $\mathbf{5 b}-\mathbf{5 m}$ were prepared and characterized as shown in Table 1 and Table 2.

\section{Experiment}

\subsection{Preparation of Ethyl 6-Bromo-2,7-Dimethylimidazo[1,2-a] Pyridine-3-Carboxylate (2a)}

A mixture of 5-bromo-4-methylpyridin-2-amine (10.0 g, $53.5 \mathrm{mmol})$ and ethyl 2-chloroacetoacetate $(8.13 \mathrm{ml}, 58.8 \mathrm{mmol})$ were taken in 1,2-dimethoxyethane $(107 \mathrm{~mL})$ and heated to reflux for 16 hours. The reaction mixture was concentrated under reduced pressure to get crude compound.

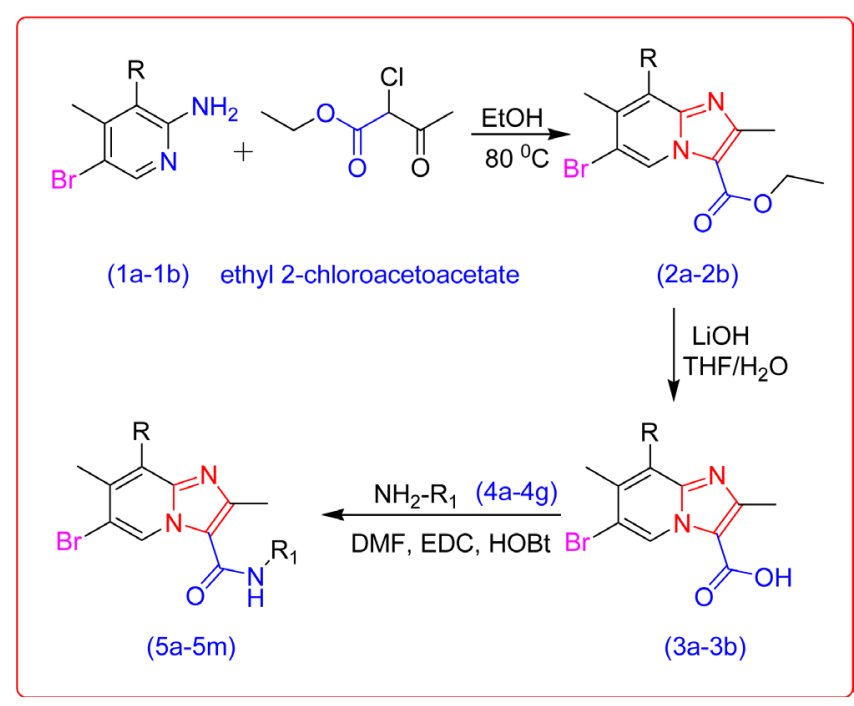

Figure 1. Reaction scheme for preparation of compounds $5 \mathrm{a}-5 \mathrm{~m}$. 
Table 1. Structures of compounds 5a-5g.

\begin{tabular}{|c|c|c|}
\hline Entry & Amine [4] & Product [5] \\
\hline a & & \\
\hline $\mathrm{b}$ & & \\
\hline c & & \\
\hline $\mathrm{d}$ & & \\
\hline $\mathrm{e}$ & & \\
\hline $\mathrm{f}$ & & \\
\hline g & $\mathrm{NH}_{2}$ & \\
\hline
\end{tabular}

Table 2. Structures of compounds $5 \mathrm{~h}-5 \mathrm{~m}$.

Entry Entry [5]

The crude compound was then purified by column chromatography using $15 \%$ ethylacetate (EtOAc) in hexane as eluent to get ethyl 6-bromo-2,7-dimethylimidazo- 
[1,2-a]pyridine-3-carboxylate (2a) (9.50 g, 66\%) as an Off-white solid; ESIMS m/z. 299 $[\mathrm{M}+2]$.

\subsection{Preparation of Ethyl 6,8-Dibromo-2,7-Dimethylimidazo[1,2-a] Pyridine-3-Carboxylate (2b)}

Compound (2b) prepared by similar process 4.1. (11.47 g, 57\%) as an off-white solid.

\subsection{Preparation of 6-Bromo-2,7-Dimethylimidazo[1,2-a] Pyridine-3-Carboxylic Acid (3a)}

A mixture of 6-bromo-2,7-dimethylimidazo[1,2-a]pyridine-3-carboxylate (9.000 g, 30.3 $\mathrm{mmol}$ ) and Lithium hydroxide (1.088 g, $45.4 \mathrm{mmol})$ were taken in THF/Water (1:1) (60 $\mathrm{mL}$ ) and stirred at room temperature for $14 \mathrm{~h}$. The reaction mixture extracted three times with $25 \mathrm{ml}$ of ethyl acetate, further organic layers were discarded. Aqueous layer acidified with $2 \mathrm{NHCl}$ at $5^{\circ} \mathrm{C}-10^{\circ} \mathrm{C}$ till the reaction mixture turned to $\mathrm{pH} \sim 5$, the precipitate formed was filtered and dried to get 6-bromo-2,7-dimethylimidazo [1,2-a]pyridine-3-carboxylic acid (3a) (7.34 g, 92\%) as an off-white solid. EIMS $m / z .271[\mathrm{M}+2]$.

\subsection{Preparation of 6,8-Dibromo-2,7-Dimethylimidazo[1,2-a] Pyridine-3-Carboxylic Acid (3b)}

Compound ( $3 \mathrm{~b}$ ) is prepared by process 4.3 . (8.35 g, $82 \%)$ as an off-white solid. EIMS $m / z: 348[\mathrm{M}+1]$.

\subsection{General Procedure for the Preparation of 6,8-Dibromo-2, 7-Dimethyl-N-Phenylimidazo[1,2-a]Pyridine-3-Carboxamides (5a-5m)}

To the stirred solution of carboxylic acid (3a-3b) (1.0 equiv), EDCI (1.2 equiv), $\mathrm{HOBt}$ (1.2 equiv) and $\mathrm{Et}_{3} \mathrm{~N}$ (2.5 equiv) in $\mathrm{DMF}$ at $0^{\circ} \mathrm{C}$, was added compound (4a-4g) (1.05 equiv) and allowed to stir at room temperature for $22 \mathrm{~h}$. The reaction mixture was diluted with dichloromethane, washed with water and the separated organic layer was concentrated under reduced pressure. The crude compound was further purified by column chromatography using $30 \%$ EtOAc/Hexanes as the eluent.

\subsubsection{Preparation of 6,8-Dibromo-2,7-Dimethyl-N-Phenylimidazo[1,2-a] Pyridine-3-Carboxamide (5a)}

The stirred solution of 6,8-dibromo-2,7-dimethylimidazo[1,2-a]pyridine-3-carboxylic acid $(0.5 \mathrm{~g}, 1.437 \mathrm{mmol})$ in DMF $(14.37 \mathrm{ml})$ at $0^{\circ} \mathrm{C}$ was mixed with EDCI $(0.331 \mathrm{~g}$, $1.724 \mathrm{mmol})$, HOBt $(0.233 \mathrm{~g}, 1.724 \mathrm{mmol})$ and Triethylamine $(0.505 \mathrm{ml}, 3.59 \mathrm{mmol})$. The reaction mixture was stirred for $30 \mathrm{~min}$ and then aniline $4 \mathrm{a}(0.138 \mathrm{ml}, 1.509 \mathrm{mmol})$ was added. The reaction mixture was allowed to stir further at room temperature for 22 $\mathrm{h}$. The reaction mixture was then diluted with dichloromethane and washed with water and the separated organic layer was concentrated under reduced pressure, purified by column chromatography using $30 \% \mathrm{EtOAc/Hexanes} \mathrm{as} \mathrm{eluent} \mathrm{to} \mathrm{get} \mathrm{title} \mathrm{compound} \mathrm{in}$ $70 \%$ yield $(0.42 \mathrm{~g})$ White solid; m.p. $=134^{\circ} \mathrm{C}-137^{\circ} \mathrm{C} ;{ }^{1} \mathrm{H}-\mathrm{NMR}\left(400 \mathrm{MHz}, \mathrm{CDCl}_{3}\right): \delta$ $2.69(\mathrm{~s}, 3 \mathrm{H}), 2.87(\mathrm{~s}, 3 \mathrm{H}), 7.22-7.18(\mathrm{~m}, 1 \mathrm{H}), 7.43-7.39(\mathrm{t}, 2 \mathrm{H}), 7.61-7.59$ (d, $J=8.0$ 
$\mathrm{Hz}, 1 \mathrm{H}), 7.69-7.78(\mathrm{~m}, 2 \mathrm{H}), 9.67(\mathrm{~s}, 1 \mathrm{H}) ;{ }^{13} \mathrm{C}-\mathrm{NMR}\left(400 \mathrm{MHz}^{-\mathrm{CDCl}_{3}}\right): \delta 16.1,22.7$, $110.3,110.9,116.5,120.0,122.4,123.1,124.2,124.5,126.5,128.8,136.8,137.3,145.5$, 158.5; IR $\left(\mathrm{cm}^{-1}\right)$ : 3448 (Aromatic C-H Stretch), 3138 (Amide N-H Stretch), 1654 (Amide $\mathrm{C}=\mathrm{O}$ Stretch), 1595 (Amide N-H bending); EIMS m/z: $423.9\left[\mathrm{M}^{+}\right]$.

4.5.2. Preparation of 6,8-Dibromo-2,7-Dimethyl-N-(4-Fluorophenyl) Imidazo[1,2-a]Pyridine-3-Carboxamide (5b)

$73 \%$ yield $(0.46 \mathrm{~g})$, White solid; m.p. $=142^{\circ} \mathrm{C}-143^{\circ} \mathrm{C} ;{ }^{1} \mathrm{H}-\mathrm{NMR}\left(400 \mathrm{MHz}, \mathrm{CDCl}_{3}\right): \delta$ $2.69(\mathrm{~s}, 3 \mathrm{H}), 2.86(\mathrm{~s}, 3 \mathrm{H}), 7.13-7.09(\mathrm{~m}, 2 \mathrm{H}), 7.48-7.50(\mathrm{~m}, 1 \mathrm{H}), 7.57$ - $7.54(\mathrm{dd}, 2 \mathrm{H})$, $9.63(\mathrm{~s}, 1 \mathrm{H}) ;{ }^{13} \mathrm{C}-\mathrm{NMR}\left(400 \mathrm{MHz}, \mathrm{CDCl}_{3}\right): \delta 16.3,22.7,110.7,111.1,115.4,115.7,116.2$, 122.0, 122.1, 123.4, 124.1, 126.2, 126.6, 145.6, 158.2, 158.6; IR $\left(\mathrm{cm}^{-1}\right): 3443$ (Aromatic C-H Stretch), 3141 (Amide N-H Stretch), 1653 (Amide C=O Stretch), 1607 (Amide $\mathrm{N}-\mathrm{H}$ bending); EIMS $m / z .441 .8\left[\mathrm{M}^{+}\right]$.

\subsubsection{Preparation of 6,8-Dibromo-N-(2,4-Difluorophenyl)-2,} 7-Dimethylimidazo[1,2-a]Pyridine-3-Carboxamide (5c)

$68 \%$ yield $(0.44 \mathrm{~g})$, White solid; m.p. $=151^{\circ} \mathrm{C}-154^{\circ} \mathrm{C} ;{ }^{1} \mathrm{H}-\mathrm{NMR}\left(400 \mathrm{MHz}, \mathrm{CDCl}_{3}\right): \delta$ $2.70(\mathrm{~s}, 3 \mathrm{H}), 2.88(\mathrm{~s}, 3 \mathrm{H}), 6.99-6.93(\mathrm{~m}, 2 \mathrm{H}), 8.40-8.34(\mathrm{~m}, 2 \mathrm{H}), 9.69(\mathrm{~s}, 1 \mathrm{H})$; IR $\left(\mathrm{cm}^{-1}\right)$ : 3292 (Aromatic C-H Stretch), 3122 (Amide N-H Stretch), 1664 (Amide C=O Stretch), 1611 (Amide N-H bending); EIMS m/z. $459.8\left[\mathrm{M}^{+}\right]$.

4.5.4. Preparation of 6,8-Dibromo-N-(3-Chlorophenyl)-2, 7-Dimethylimidazo[1,2-a]Pyridine-3-Carboxamide (5d)

$69 \%$ yield $(0.45 \mathrm{~g})$, White solid; M.P $=161^{\circ} \mathrm{C}-163^{\circ} \mathrm{C} ;{ }^{1} \mathrm{H}-\mathrm{NMR}\left(400 \mathrm{MHz}, \mathrm{CDCl}_{3}\right): \delta$ $2.70(\mathrm{~s}, 3 \mathrm{H}), 2.87(\mathrm{~s}, 3 \mathrm{H}), 7.19-7.16(\mathrm{~m}, 2 \mathrm{H}), 7.44-7.42(\mathrm{~m}, 2 \mathrm{H}), 7.76-7.75(\mathrm{t}, 1 \mathrm{H})$, 9.66 (s, 1H); IR ( $\left.\mathrm{cm}^{-1}\right): 3413$ (Aromatic C-H Stretch), 3129 (Amide N-H Stretch), 1651 (Amide $\mathrm{C}=\mathrm{O}$ Stretch), 1587 (Amide N-H bending); EIMS m/z: $457.8\left[\mathrm{M}^{+}\right]$.

\subsubsection{Preparation of 6,8-Dibromo-N-(3-Chloro-4-Fluorophenyl)-2,} 7-Dimethylimidazo[1,2-a]Pyridine-3-Carboxamide (5e)

$61 \%$ yield $(0.41 \mathrm{~g})$ White solid; m.p. $=146^{\circ} \mathrm{C}-148^{\circ} \mathrm{C} ;{ }^{1} \mathrm{H}-\mathrm{NMR}\left(400 \mathrm{MHz}, \mathrm{CDCl}_{3}\right): \delta$ $2.70(\mathrm{~s}, 3 \mathrm{H}), 2.86(\mathrm{~s}, 3 \mathrm{H}), 7.20-7.15(\mathrm{t}, 1 \mathrm{H}), 7.42-7.38(\mathrm{~m}, 2 \mathrm{H}), 7.82-7.80(\mathrm{dd}, 1 \mathrm{H})$, $9.63(\mathrm{~s}, 1 \mathrm{H}) ; \mathrm{IR}\left(\mathrm{cm}^{-1}\right): 3426$ (Aromatic C-H Stretch), 3129 (Amide N-H Stretch), 1650 (Amide $\mathrm{C}=\mathrm{O}$ Stretch), 1500 (Amide N-H bending); EIMS m/z: $475.8\left[\mathrm{M}^{+}\right]$.

\subsubsection{Preparation of 6,8-Dibromo-N-(2,5-Dimethylphenyl)-2,} 7-Dimethylimidazo[1,2-a]Pyridine-3-Carboxamide (5f)

$70 \%$ yield $(0.45 \mathrm{~g})$ White solid; m.p. $=159^{\circ} \mathrm{C}-163^{\circ} \mathrm{C} ;{ }^{1} \mathrm{H}-\mathrm{NMR}\left(400 \mathrm{MHz}, \mathrm{CDCl}_{3}\right): \delta$ $2.32(\mathrm{~s}, 3 \mathrm{H}), 2.38(\mathrm{~s}, 3 \mathrm{H}), 2.69(\mathrm{~s}, 3 \mathrm{H}), 2.88(\mathrm{~s}, 3 \mathrm{H}), 6.97$ - $6.95(\mathrm{~d}, 1 \mathrm{H}), 7.15$ - $7.13(\mathrm{~m}$, 2H), 7.83 (s, 1H), $9.72(\mathrm{~s}, 1 \mathrm{H})$; IR $\left(\mathrm{cm}^{-1}\right)$ : 3452 (Aromatic C-H Stretch), 3127 (Amide $\mathrm{N}-\mathrm{H}$ Stretch), 1651 (Amide C=O Stretch), 1580 (Amide N-H bending); EIMS $\mathrm{m} / \mathrm{z}$. $451.9\left[\mathrm{M}^{+}\right]$.

4.5.7. Preparation of 6,8-Dibromo-2,7-Dimethyl-N-(1-Phenylethyl)Imidazo [1,2-a]Pyridine-3-Carboxamide (5g)

$68 \%$ yield $\left(0.44\right.$ g) White solid; m.p. $=132^{\circ} \mathrm{C}-134^{\circ} \mathrm{C} ;{ }^{1} \mathrm{H}-\mathrm{NMR}\left(400 \mathrm{MHz}, \mathrm{CDCl}_{3}\right): \delta$ 
$1.65-1.64(\mathrm{t}, 3 \mathrm{H}), 2.66(\mathrm{~s}, 3 \mathrm{H}), 2.73(\mathrm{~s}, 3 \mathrm{H}), 5.36-5.29(\mathrm{q}, 2 \mathrm{H}), 7.41-7.28(\mathrm{~m}, 5 \mathrm{H})$, 9.64 (s, 1H); IR ( $\left.\mathrm{cm}^{-1}\right)$ : 3451 (Aromatic C-H Stretch), 3125 (Amide N-H Stretch), 1655 (Amide C=O Stretch); EIMS m/z: $451.9\left[\mathrm{M}^{+}\right]$.

\subsubsection{Preparation of 6-Bromo-2,7-Dimethyl-N-Phenylimidazo[1,2-a]} Pyridine-3-Carboxamide (5h)

$54 \%$ yield $(0.34 \mathrm{~g})$ as white solid; m.p. $=121^{\circ} \mathrm{C}-123{ }^{\circ} \mathrm{C} ;{ }^{1} \mathrm{H}-\mathrm{NMR}\left(400 \mathrm{MHz}, \mathrm{CDCl}_{3}\right): \delta$ $2.48(\mathrm{~s}, 3 \mathrm{H}), 2.81(\mathrm{~s}, 3 \mathrm{H}), 7.20-7.16(\mathrm{t}, 1 \mathrm{H}), 7.44-7.37(\mathrm{~m}, 3 \mathrm{H}), 7.48$ (brs, 1H), 7.61 $7.58(\mathrm{~d}, 2 \mathrm{H}), 9.64$ (s, 1H); IR $\left(\mathrm{cm}^{-1}\right): 3305$ (Aromatic C-H Stretch), 3044 (Amide N-H Stretch), 1625 (Amide $\mathrm{C}=\mathrm{O}$ Stretch), 1597 (Amide $\mathrm{N}-\mathrm{H}$ bending); EIMS m/z: 346.20 $[\mathrm{M}+2]$.

\subsubsection{Preparation of 6-Bromo-N-(4-Fluorophenyl)-2,7-Dimethyl} Imidazo[1,2-a]Pyridine-3-Carboxamide (5i)

$64 \%$ yield (0.43 g), White solid; m.p. $=144^{\circ} \mathrm{C}-146^{\circ} \mathrm{C} ;{ }^{1} \mathrm{H}-\mathrm{NMR}\left(400 \mathrm{MHz}, \mathrm{CDCl}_{3}\right): \delta$ $2.50(\mathrm{~s}, 3 \mathrm{H}), 2.82(\mathrm{~s}, 3 \mathrm{H}), 7.12-7.08(\mathrm{~m}, 2 \mathrm{H}), 7.51(\mathrm{~s}, 1 \mathrm{H}), 7.60-7.56(\mathrm{~m}, 2 \mathrm{H}), 7.70$ (brs, $1 \mathrm{H}), 9.60(\mathrm{~s}, 1 \mathrm{H}) ;{ }^{13} \mathrm{C}-\mathrm{NMR}\left(400 \mathrm{MHz}, \mathrm{CDCl}_{3}\right): \delta 16.3,22.5,112.3,115.1,115.4$, 121.6, 122.0, 127.8, 138.5, 158.1, 158.8, 160.5; IR $\left(\mathrm{cm}^{-1}\right)$ : 3292 (Aromatic C-H Stretch), 3043 (Amide N-H Stretch), 1625 (Amide C=O Stretch); EIMS m/z. 364.0 [M + 2].

\subsubsection{Preparation of 6-Bromo-N-(3-Chlorophenyl)-2,7-Dimethyl} Imidazo[1,2-a]Pyridine-3-Carboxamide (5j)

$62 \%$ yield $(0.45 \mathrm{~g})$, as white solid; m.p. $=144^{\circ} \mathrm{C}-146{ }^{\circ} \mathrm{C} ;{ }^{1} \mathrm{H}-\mathrm{NMR}\left(400 \mathrm{MHz}, \mathrm{CDCl}_{3}\right): \delta$ $2.50(\mathrm{~s}, 3 \mathrm{H}), 2.82(\mathrm{~s}, 3 \mathrm{H}), 7.17-7.15(\mathrm{~d}, 1 \mathrm{H}), 7.34-7.30(\mathrm{t}, 1 \mathrm{H}), 7.49-7.43(\mathrm{~m}, 3 \mathrm{H})$, 7.76 - 7.76 (m, 1H), 9.65 (s, 1H); IR $\left(\mathrm{cm}^{-1}\right)$ : 3326 (Aromatic C-H Stretch), 3025 (Amide $\mathrm{N}-\mathrm{H}$ Stretch), 1632 (Amide $\mathrm{C}=\mathrm{O}$ Stretch), 1589 (Amide N-H bending); EIMS $\mathrm{m} / z$. $379.9[\mathrm{M}+2]$.

\subsubsection{Preparation of 6-Bromo-N-(3-Chloro-4-Fluorophenyl)-2,} 7-Dimethylimidazo[1,2-a]Pyridine-3-Carboxamide (5k)

$62 \%$ yield $(0.45 \mathrm{~g})$, White solid; m.p. $=136^{\circ} \mathrm{C}-138^{\circ} \mathrm{C} ;{ }^{1} \mathrm{H}-\mathrm{NMR}\left(400 \mathrm{MHz}, \mathrm{CDCl}_{3}\right): \delta 2.50$ (s, 3H), $2.82(\mathrm{~s}, 3 \mathrm{H}), 7.19-7.15(\mathrm{t}, 1 \mathrm{H}), 7.42$ - $7.38(\mathrm{~m}, 2 \mathrm{H}), 7.47(\mathrm{t}, 1 \mathrm{H}), 7.82$ - 7.79 (dd, 1H), 9.64 (s, 1H); IR ( $\left.\mathrm{cm}^{-1}\right): 3336$ (Aromatic C-H Stretch), 3027 (Amide N-H Stretch), 1630 (Amide C=O Stretch), 1599 (Amide N-H bending); EIMS m/z. 397.9 [M + 2].

4.5.12. Preparation of 6-Bromo-N-(2,5-Dimethylphenyl)-2, 7-Dimethylimidazo[1,2-a]Pyridine-3-Carboxamide (51) $66 \%$ yield $(0.45 \mathrm{~g})$ White solid; m.p. $=151^{\circ} \mathrm{C}-154{ }^{\circ} \mathrm{C} ;{ }^{1} \mathrm{H}-\mathrm{NMR}\left(400 \mathrm{MHz}, \mathrm{CDCl}_{3}\right): \delta$ $2.32(\mathrm{~s}, 3 \mathrm{H}), 2.41(\mathrm{~s}, 3 \mathrm{H}), 2.50(\mathrm{~s}, 3 \mathrm{H}), 2.82(\mathrm{~s}, 3 \mathrm{H}), 7.19-7.15(\mathrm{t}, 1 \mathrm{H}), 7.42$ - $7.38(\mathrm{~m}$, $2 \mathrm{H}), 7.47(\mathrm{t}, 1 \mathrm{H}), 7.82-7.79(\mathrm{dd}, 1 \mathrm{H}), 9.64(\mathrm{~s}, 1 \mathrm{H}) ; \mathrm{IR}\left(\mathrm{cm}^{-1}\right): 3453$ (Aromatic C-H Stretch), 3135 (Amide N-H Stretch), 1644 (Amide C=O Stretch), 1582 (Amide N-H bending); EIMS $m / z: 374.0[\mathrm{M}+2]$.

\subsubsection{Preparation of 6-Bromo-2,7-Dimethyl-N-(1-Phenylethyl)Imidazo}

[1,2-a]Pyridine-3-Carboxamide (5m)

$64 \%$ yield $(0.44 \mathrm{~g})$, White solid; m.p. $=181^{\circ} \mathrm{C}-186^{\circ} \mathrm{C} ;{ }^{1} \mathrm{H}-\mathrm{NMR}\left(400 \mathrm{MHz}, \mathrm{CDCl}_{3}\right): \delta$ 
$1.65-1.63(\mathrm{~d}, 3 \mathrm{H}), 2.48(\mathrm{~s}, 3 \mathrm{H}), 2.69(\mathrm{~s}, 3 \mathrm{H}), 5.37-5.30(\mathrm{q}, 1 \mathrm{H}), 6.01-5.99(\mathrm{~d}, 1 \mathrm{H})$, 7.42 - $7.28(\mathrm{~m}, 6 \mathrm{H}), 9.64(\mathrm{~s}, 1 \mathrm{H})$; IR $\left(\mathrm{cm}^{-1}\right): 3317$ (Aromatic C-H Stretch), 3027 (Amide N-H Stretch), 1615 (Amide C=O Stretch); EIMS m/z. 374.0 [M + 2].

\section{Results and Discussion}

\section{Antimycobacterial activity}

The anti-tubercular activity of the synthesized compounds (5a-5m) against $\mathrm{Myco}$ bacterium tuberculosis (H37 RV strain) ATCC No-27294, were assessed at the Department of Microbiology, Maratha Mandal's NGH Institute of Dental Sciences and Research Centre, Belgaum-590010, India. The method applied is similar to that reported by Maria and Lourenco [23].

The M. Tuberculosis stain H37Rv (ATCC 27294) was cultured at $37^{\circ} \mathrm{C}$ in LowesteinJensenn medium until log phase growth. Then a cell suspension was prepared at a concentration of about $2 \times 10^{6} \mathrm{UFC}$ Ml-CM and further diluted 1:20 in Middlebrook 7H9 medium. The later was supplemented with 10\% OADC (oleic acid-albumin-dextrose-catalase) and $0.001 \%$ Tween 80 . One $\mathrm{mL}$ bactarial suspention was added to each tube (Capped, glass) to gather with the sample solutions of various concentrations. The final concentrations of the compounds under test ranged from 0.8 to $100 \mu \mathrm{g}$ per $\mathrm{mL}$ and adjusted to a final $2 \mathrm{~mL}$ volume. After a 7 day incubation $100 \mu \mathrm{l}$ of 3-(4,5-dimethylthiazol-2-yl)-2,5-diphenyltetrazolium bromide (MTT) $(5 \mathrm{mg} / \mathrm{mL})$ with $20 \%$ Tween 80 was added to the glass tubes. A violet colour indicated bacterial growth. The tubes were evaluated for colour change on day 8. For standard tests MIC value of Pyrazinamide-3.125 $\mu \mathrm{g} / \mathrm{mL}$, Streptomycin-6.25 $\mu \mathrm{g} / \mathrm{mL}$ and Ciprofloxacin-3.125 $\mu \mathrm{g} / \mathrm{mL}$, were determined each time. The MIC of each sample corresponded to the lowest concentration at which the bacteria tested did not show growth. Susceptibility testing was performed three times. The results were expressed as the mean of three tests.

However, compounds $5 \mathrm{a}-\mathbf{5 m}$ showed moderate to good anti-tubercular activity as represented in Figure 2. This is due to the presence of active structural moieties like imidazo[1,2-a]pyridine and carboxamide group, which might interfere in the mechanism of cell mitosis and hence stop further growth of Mycobacterium tuberculosis. All the studied samples are showing dissimilar potency due to the effective barrier of cell wall membrane of Mycobacterium tuberculosis for entrance of external substances like test compounds under this study. In addition, these compounds disturb the respiration process of the cell and thereby restrict the biosynthesis of proteins. If the biosynthesis of proteins is blocked then formation of bacterial cell wall is not possible which ultimately results in cell death and therefore restricts further growth and infection due to bacteria [24].

\section{Conclusion}

In conclusion, new derivatives of imidazo[1,2-a]pyridine have been synthesized successfully with good yield. The experimental data for the evaluation of anti-tuberculosis activity revealed that most of the synthesized compounds $5 \mathrm{a}-5 \mathrm{~m}$ demonstrated 


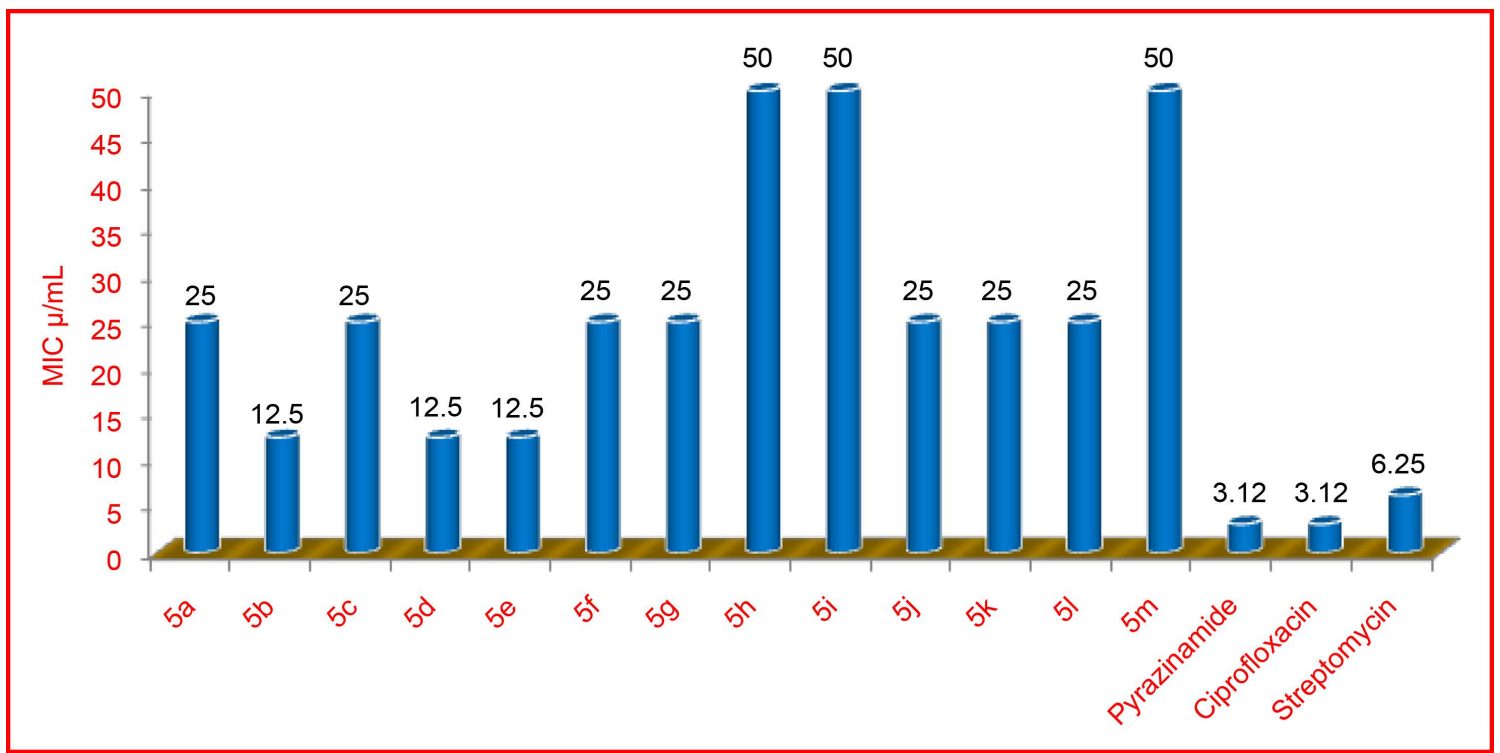

Figure 2. MIC in $\mu \mathrm{g} / \mathrm{mL}$ for test samples $5 \mathrm{a}-5 \mathrm{~m}$.

moderate to good antituberculosis activity. Among the tested compounds $5 \mathrm{~b}, 5 \mathrm{~d}$ and $5 \mathrm{e}$ were found to be the most active with minimum inhibitory concentration (MIC) of 12.5 $\mu \mathrm{g} / \mathrm{mL}$ against Mycobacterium tuberculosis (H37 RV strain) ATCC No-27294 with minimum inhibitory concentration of $12.5 \mu \mathrm{g} / \mathrm{ml}$. This study would pave the way for future development of more effective imidazo[1,2-a]pyridine analogs for applications in biological science.

\section{Acknowledgements}

The authors thank Principal and Head Department of Chemistry, Government of Maharashtra, Ismail Yusuf Arts, Science and Commerce College for providing research and library facilities. The authors also thank Management and Principal of C. S.'s Patkar-Varde College, Goregaon (W), Mumbai for their constant encouragement and support. The authors also acknowledge the help of Dr. Kishore Bhat of Governmental Dental College, Belgaum, for facilitating anti-TB assays and providing the procedure for the same.

\section{References}

[1] Katritzky, A., Xu, Y. and Tu, H. (2003) Regiospecific Synthesis of 3-Substituted Imidazo[1,2-a]Pyridines, Imidazo[1,2-a]Pyrimidines, and Imidazo[1,2-c]Pyrimidine. The Journal of Organic Chemistry, 68, 4935-4937. https://doi.org/10.1021/jo026797p

[2] Rival, Y., Grassy, G. and Michel, G. (1992) Synthesis and Antibacterial Activity of Some Imidazo[1,2-a]Pyrimidine Derivatives. Chemical and Pharmaceutical Bulletin, 40, 11701176. https://doi.org/10.1248/cpb.40.1170

[3] Moraski, G., Garrett, C., Lowell, M., Jeffrey, C., Philip, H., Helena, B., Mai, B., Torey, A., Juliane, O., Tanya, P. and Marvin, M. (2013) Advancement of Imidazo[1,2-a]Pyridines with Improved Pharmacokinetics and nM Activity vs. Mycobacterium tuberculosis. ACS Medicinal Chemistry Letters, 4, 675-679. https://doi.org/10.1021/ml400088y 
[4] Rival, Y., Grassy, G., Taudou, A. and Ecalle, R. (1991) Antifungal Activity in Vitro of Some Imidazo[1,2-a]Pyrimidine Derivatives. European Journal of Medicinal Chemistry, 26, 1318. https://doi.org/10.1016/0223-5234(91)90208-5

[5] Chaouni-Bendallah, A., Galtier, C., Allouchi, H. and Kherbeche, A. (2001) 3-Benzamido, Ureido and Thioureidoimidazo[1,2-a]Pyridine Derivatives as Potential Antiviral Agents. Chemical and Pharmaceutical Bulletin, 49, 1631-1635. https://doi.org/10.1248/cpb.49.1631

[6] Chezal, J., Paeshuyse, J., Gaumet, V., Canitrota, D., Maisonial, A., Lartigue, C., Gueiffier, A., Moreaua, E., Teulade, J., Chavignon, O. and Neyts, J. (2010) Synthesis and Antiviral Activity of an Imidazo[1,2-a]Pyrrolo[2,3-c]Pyridine Series against the Bovine Viral Diarrhea Virus. European Journal of Medicinal Chemistry, 45, 2044-2047.

https://doi.org/10.1016/j.ejmech.2010.01.023

[7] Sanfilippo, P.J., Urbanski, M., Press, J.B. and Moore, J.B.J. (1998) Synthesis of (Aryloxy)Alkylamines. 2. Novel Imidazo-Fused Heterocycles with Calcium Channel Blocking and Local Anesthetic Activity. Journal of Medicinal Chemistry, 31, 2221-2227. https://doi.org/10.1021/jm00119a026

[8] Byth, K.F., Culshaw, J.D., Green, S., Oakes, S. and Thomaset, A.P. (2004) Imidazo[1,2-a]Pyridines. Part 2: SAR and Optimisation of a Potent and Selective Class of Cyclin-Dependent Kinase Inhibitors. Bioorganic \& Medicinal Chemistry Letters, 14, 2245-2248. https://doi.org/10.1016/j.bmcl.2004.02.015

[9] Dvey, D., Erhardt, P.W., Lumma, W.C. and Cantor, E. (1987) Cardiotonic Agents. 1. Novel 8-aryl Substituted Imidazo[1,2-a]- and -[1,5-a]Pyridines and Imidazo[1,5-a]Pyridinones as Potential Positive Inotropic Agents. Journal of Medicinal Chemistry, 30, 1337-1342. https://doi.org/10.1021/jm00391a012

[10] Kaminnski, J.J. and Doweyko, A.M. (1997) Antiulcer Agents. 6. Analysis of the in Vitro Biochemical and in Vivo Gastric Antisecretory Activity of Substituted Imidazo[1,2-a]-pyridines and Related Analogues Using Comparative Molecular Field Analysis and Hypothetical Active Site Lattice Methodologies. Journal of Medicinal Chemistry, 40, 427-436. https://doi.org/10.1021/jm950700s

[11] Zhonghui, L., Gregory, R., Anand, R. and Rui-Qin, L. (2008) Potent, Selective, Orally Bioavailable Inhibitors of Tumor Necrosis Factor- $\alpha$ Converting Enzyme (TACE): Discovery of Indole, Benzofuran, Imidazopyridine and Pyrazolopyridine P1' Substituents. Bioorganic \& Medicinal Chemistry Letters, 18, 1958-1962. https://doi.org/10.1016/j.bmcl.2008.01.120

[12] Gregory, R., Anand R., Zhonghui, L. and Rui-Qin, L. (2008) Potent, Exceptionally Selective, Orally Bioavailable Inhibitors of TNF- $\alpha$ Converting Enzyme (TACE): Novel 2-Substituted-1 $H$-Benzo[ $d$ ]Imidazol-1-yl)Methyl)Benzamide P1' Substituents. Bioorganic \& Medicinal Chemistry Letters, 18, 1577-1582. https://doi.org/10.1016/j.bmcl.2008.01.075

[13] Moraski, G.C., Miller, P.A., Bailey M.A., Ollinger, J., Parish, T., Boshoff, H.I., Cho, S., Anderson, J.R., Mulugeta, S., Franzblau, S.G. and Miller, M.J. (2015) Putting Tuberculosis (TB) to Rest: Transformation of the Sleep Aid, Ambien, and "Anagrams" Generated Potent Antituberculosis Agents. ACS Infectious Diseases, 13, 85-90. https://doi.org/10.1021/id500008t

[14] Abrahams, K.A., Jonathan A., Cox, G., Vickey, L., Loman, N.J., Pallen, M.J., Constantinidou, C., Fernández, R., Alemparte, C., Remuiñán, M.J., Barros, D., Ballell, L. and Besra, G.S. (2012) Identification of Novel Imidazo[1,2- $\alpha$ ]Pyridine Inhibitors Targeting M. tuberculosis QcrB. PLoS ONE, 7, e52951. https://doi.org/10.1371/journal.pone.0052951

[15] Moraski, G.C., Markley, L.D., Hipskind, P.A., Boshoff, H., Cho, S., Franzblau, S.G. and Miller, M.J. (2011) Advent of Imidazo[1,2- $\alpha$ ]Pyridine-3-Carboxamides with Potent Multiand Extended Drug Resistant Antituberculosis Activity. ACS Medicinal Chemistry Letters, 2, 466-470. https://doi.org/10.1021/ml200036r 
[16] Li, L., Li, Z., Liu, M., Shen, W., Wang, B., Guo, H. and Lu, Y. (2016) Design, Synthesis and Antimycobacterial Activity of Novel Imidazo[1,2- $\alpha$ ]Pyridine Amide-Cinnamamide Hybrids. Molecules, 21, 49. https://doi.org/10.3390/molecules21010049

[17] Samala, G., Nallangi, R., Devi, P.B., Saxena, S., Yadav, R., Sridevi, J.P., Yogeeswari, P. and Sriram, D. (2014) Identification and Development of 2-Methylimidazo[1,2- $\alpha]$ Pyridine -3-Carboxamides as Mycobacterium tuberculosis Pantothenate Synthetase Inhibitors. Bioorganic \& Medicinal Chemistry, 22, 4223-4232. https://doi.org/10.1016/j.bmc.2014.05.038

[18] Mandewale, M.C., Thorat, B.R., Shelke, D. and Yamgar, R.S. (2015) Synthesis and Biological Evaluation of New Hydrazone Derivatives of Quinoline and Their $\mathrm{Cu}$ (II) and $\mathrm{Zn}$ (II) Complexes against Mycobacterium tuberculosis. Bioinorganic Chemistry and Applications, 2015, Article ID: 153015. https://doi.org/10.1155/2015/153015

[19] Mandewale, M.C., Thorat, B.R., Nivid, Y., Jadhav, R., Nagarsekar, A.S. and Yamgar, R.S. (2016) Synthesis, Structural Studies and Antituberculosis Evaluation of New Hydrazone Derivatives of Quinoline and Their Zn(II) Complexes. Journal of Saudi Chemical Society, In Press. https://doi.org/10.1016/j.jscs.2016.04.003

[20] Mandewale, M.C., Kokate, S., Thorat, B.R., Sawant, S.S. and Yamgar, R.S. (2016) Zinc Complexes of Hydrazone Derivatives Bearing 3,4-Dihydroquinolin-2(1h)-One Nucleus as New Anti-Tubercular Agents. Arabian Journal of Chemistry, In Press. https://doi.org/10.1016/j.arabjc.2016.07.016

[21] Mandewale, M.C., Thorat B.R. and Yamgar, R.S. (2015) Synthesis and Anti-Mycobacterium Study of Some Fluorine Containing Schiff Bases of Quinoline and Their Metal Complexes. Der Pharma Chemica, 7, 207-215.

[22] Nivid, Y., Jadhav, B.S., Kenny, R.S., Nazirkar, B.P., Thorat, B.R., Mulgoankar, B.S. and Yamgar, R.S. (2015) Synthesis and Biological Activity Studies of Some Novel Substituted Imidazo[1,2-A]Pyridine. Heterocyclic Letters, 5, 177-184.

[23] Lourenco, M.S., DeSouza, M.N., Pinheiro, A.C., Ferreira, M.L., Goncalves, R.B., Nogneira T.M. and Peralta, M.A. (2007) Evaluation of Anti-Tubercular Activity of Nicotinic and Isoniazid Analogues. Arkivoc. 15, 181-191.

[24] Raman, N. (2005) Antibacterial Study of the Mannich Base, N-(1-Morpholino-Benzyl) Semicarbazide and Its Transition Metal(II) Complexes. Research Journal of Chemistry and Environment, 4, 9.

\section{Submit or recommend next manuscript to SCIRP and we will provide best service for you:}

Accepting pre-submission inquiries through Email, Facebook, LinkedIn, Twitter, etc.

A wide selection of journals (inclusive of 9 subjects, more than 200 journals)

Providing 24-hour high-quality service

User-friendly online submission system

Fair and swift peer-review system

Efficient typesetting and proofreading procedure

Display of the result of downloads and visits, as well as the number of cited articles

Maximum dissemination of your research work

Submit your manuscript at: http://papersubmission.scirp.org/

Or contact ojmc@scirp.org 